\title{
Surface-enhanced Raman Spectroscopy of Benzimidazolic Fungicides: Benzimidazole and Thiabendazole
}

\author{
Mak Soon Kim, Min Kyung Kim, Chul Jae Lee, ${ }^{\ddagger}$ Young Mee Jung, ${ }^{\ddagger}$ and Mu Sang Lee* \\ Department of Chemistry Education, Kythgpook National Lniversity, Daegu 702-701, Korea. "E-mail:mstee aknuackr \\ ${ }^{\dagger}$ Division of Chemical Industrv, Yeungham College of Science \& Technologv. Daegu 705-703, Korea \\ ${ }^{\ddagger}$ Department of Chemistry, Kangwon National University, Chunchon 200-701, Korea \\ Received September 2, 2009. Accepted October 7. 2009
}

\begin{abstract}
Surface-erhanced Raman Scattering (SERS) spectroscopy is applied to the study of the adsorption of benzoinidazolic fingicides benzimidazole (BIZ) and thiabendazole (TBZ) on silver mirrors. The influence of $\mathrm{pH}$ on the adsorption mechanism was investigated. In case of BIZ, two different adsorption mechanisms are deduced depending on the experimental conditions: via the $\pi$ electrons of the ring in neutral conditions and through an ionic pairing of protonated nitrogen atom with the chloride adsorbed on the metal surface. The SERS spectra of TBZ revealed that most molecules were adsorbed on silver surface by the $\pi$ electrons in neutral and acidic conditions but in acid conditions, sone nolecules were adsorbed via the sulfur and nitrogen atoms tilted slightly to the surface.
\end{abstract}

Key Words: Surface-enhanced Raman spectroscopy. Fungicide, Benzimidazole. Thiabendazole

\section{Introduction}

Thiabendazole (TBZ. 4-(1H-1.3-benzodiazol-2-yl)-1.3-thiazole) which belongs to the benzimidazole derivative. is a fungicide and parasiticide. It is known by the brand names Mintezol. Tresaderm, and Acrobetec. It is used primarily to control mold blight and other fungally caused diseases in frnits (e.g. oranges) and vegetables. TBZ is also used as a food additive, a preservative. For example. it is applied to bananas to ensure freshness. and is a common ingredient in the waves applied to the skin of citrus fruits. Medicinally. thiabendazole is also a chelating agent. which means that it is used medicinally to bind metals in case of metal poisoning or antimony poisoning. As an antiparasitic. it is able to control roundworms. hookworms. and other helminth species which attack wild animals, livestock and humans. ${ }^{1.3}$ Benzimidazole (BIZ, $1 / H$-benzimidazole) also is known for commercial and biological importance as pharmaceuticals and fungicides. Despite its extensive using in the fields indicated above. it is surprising the fact that there is non complete vibrational assignument for the molecule in the scientific literatures. The only vibrational spectroscopic studies are limited to a brief report of the infrared spectnum. ${ }^{4-8}$

Surface-enhanced Raman spectroscopy (SERS) is a very useful technique in the analysis of the adsorption of molecules on metal surfaces. The enhancement of the Raman signal induced by metal nanoparticles. which is the basis of SERS spectroscopy. can be understood as a contribution from two different mechanisms: electromagnetic and charge-transfer mechanisms. ${ }^{9-12}$ In the electromagnetic mechanism. the intensity of the surface plasmon resonance of the metal depends on many factors including the wavelength of the incident light and the morphology of the metal surface. ${ }^{11}$ The role of these nanoparticles is correlated with the mechanism involved in the SERS enhancement. being essential the formation of surface nanoparticles to induce a huge intensification of Raman emission through localized plasmon resonance in order to obtain high quality
SERS spectra. ${ }^{13.16}$ Recently, Saito et al. ${ }^{17}$ have shown that the using of silver mirror reaction produced a thin silver islands film on silicon wafer, which shows an order of magnitude superior surface enhancement property when conpared to a conventional SERS substrate made by vacuum evaporation. Our group showed that the silver nurror substrates have an excellent surface enhancement on the Raman signal. 18.19

Therefore. in this paper, we studied the adsorption geometry of BLZ and TBZ on silver mirrors by SERS technique. The SERS study was done by testing the influence of $\mathrm{pH}$. In order to assist in the interpretation of the Raman spectra. we have obtained preliminary assignments by means of the program HyperChem (release 7.0). PM3 Hamiltonian was used to calculate the Raman frequencies of $\mathrm{BIZ}$ and $\mathrm{TBZ}$ in this program.

\section{Experimental Section}

Analytical-reagent grade BIZ, TBZ, silver nitrate, chloric acid, sodium hydroxide. d-glucose and ammonia water were purchased from Aldrich $\mathrm{Co}$. and used without further purification. All solutions were prepared with triply distilled water and methanol. Silver mirror substrate was prepared by the Tollen's method, which is widely used for the identification of aldelyde. A glass plate of $25 \mathrm{~mm} \times 10 \mathrm{~mm} \times 1 \mathrm{~mm}$ was put in a culture dish. A $10 \mathrm{~mL}$ of $5 \%$-silver anmononia solution and $5 \mathrm{~mL}$ of $10 \%$-sodium hydroxide solution were mixed in the culture dish. Then. $10 \mathrm{~mL}$ of $5 \%$-D-glucose was added to the mixing solution. A few minutes later. the color of the solution turned to yellow, then dark brown. In the meanwhile, silver ions were reduced and deposited onto the glass plate to form a fine silver film called a silver mirror. After withdrawing the silver nurror from a culture dish it was washed with distilled water and dried in room temperature. To make use of the prepared mirror. a $1 \mu \mathrm{L}$ of $1.0 \times 10^{-3} \mathrm{M}$ BIZ or TBZ solution was dropped onto it. After the evaporation of the solvents, the substrate was ready for SERS analysis. The $\mathrm{pH}$ of the solutions 
was adjusted by injecting the necessary amounts of reagent grade $\mathrm{HCl}$ and $\mathrm{NaOH}$ solutions.

Raman and SERS spectra were recorded on a Nicolet Almega XR spectrophotometer in combination with a Thermo Scientific DXR Raman microscope by using radiation at $780 \mathrm{~nm}$ from diode laser for excitation. The laser power and confocal pinhole size were $100 \mathrm{~mW}$ and $25 \mu \mathrm{m}$. respectively. The images of the Scanuing electron microscope (SEM) were obtained with a Hitachi S- +200

\section{Results and Discussion}

As shown in Figure 1, the molecules of BIZ and TBZ are planar structures. Generally. the lone-pair electron and aromatic $\pi$ st'stem were easily bound to a metal surface. Hence. we assumed that BIZ and TBZ could be adsorbed on metal surface by the sulfur, the nitrogen atom or the $\pi$ electrons of the ring. The SEM image of silver mirror substrate prepared by Tollen's method is shown in Figure 2

Figure 3 shows the surface-enhanced Raman spectrum of BIZ adsorbed on silver mirror(a) and the ordinary Raman spectrum of BIZ in solid state(b) and 0. $1 \mathrm{M}$ solution(c). It is obvious that there are great-enhanced effects in the SER spectrum of $\mathrm{BIZ}$ on the roughened silver surface. The stronger Raman bands at 553.782 and $1012 \mathrm{~cm}^{-1}$ in SERS are assigned to ò $(\mathrm{N}-\mathrm{H})$ oop. $\delta(\mathrm{C}-\mathrm{H})$ oop and $\bar{o}(\mathrm{C}-\mathrm{C}-\mathrm{C})$ oop. respectively. The weaker band at $1279 \mathrm{~cm}^{-1}$ is assigned $v_{\text {run }}$ and $\delta(\mathrm{C}-\mathrm{H})$ ip. The band frequencies and their assignments based on literature and calculation are given in Table $1 .^{\text {-1 }}$ : To perform the PM3 calculations. we focused on the vibrational modes of BIZ. Figure 4 presents a diagram of the normal mode frequency of 553,782. 1012 and $1363 \mathrm{~cm}^{-1}$. As is well known that if the adsorption occurs via donation of $\pi$-sy stem electrons. the plane of the aromatic ring would lie parallel to the surface and the totally symmetric in-plane stretching modes would be expected to show little or no surface enhancement. and certain out-of-plane vibrations would then show enhancement as per allowance of the symmetry. This line of approach has been successfully applied by many investigators. ${ }^{23: 24}$ Besides. the $\mathrm{C}-\mathrm{H}$ stretching band at $3072 \mathrm{~cm}^{-1}$ in ordinary Raman spectrum of BIZ became weaker in SERS remarkably. Therefore. it is thought that BIZ is adsorbed on to the silver surface with its molecular plane lying flat. as is shown in Figure 6a.

In Figure 5, the SER spectra of BIZ at $\mathrm{pH} 2.0 .7 .0$ and 12.0 are shown in the wavenumber range $200 \sim 1800 \mathrm{~cm}^{-1}$ and nearby $3000 \mathrm{~cm}^{-1}$. The spectral pattern of the acidic solution spectnum differs from that of the neutral and basic solutions. The significant change in acidic condition is the intensity increase of the 628,942 . and $117+\mathrm{cm}^{-1}$ modes. Also. the 1353,1513 and $1624 \mathrm{~cm}^{.1}$ modes are enhanced. The discussion about the orientation of the BIZ molecules to the silver surface has been made by applying the results of "surface selection rules" in the inplane and out-of-plane vibrations. Therefore according to these observations mentioned above in Figure 5 . it is thought that $\mathrm{BIZ}$ is adsorbed on to the silver surface via the nitrogen atom with its molecular plane standing perpendicularly. These adsorptions are possible by the formation of a strong ionic pair a nitrogen atom with the anion (in this case chloride) previously (a)

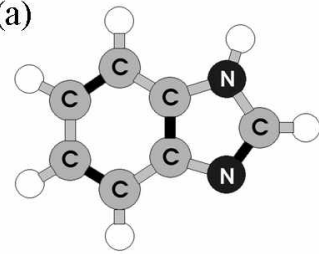

(b)

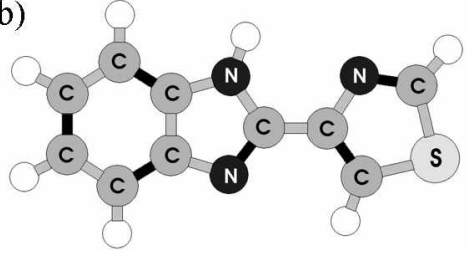

Figure 1. Chemical structure of the fungicides: (a) benzimidazole and (b) thiabendazole.

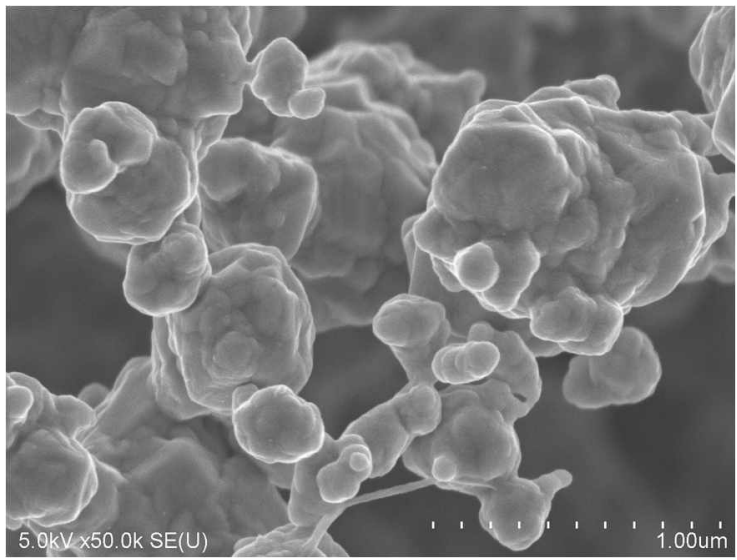

Figure 2. The SEM inage of silver mirror.

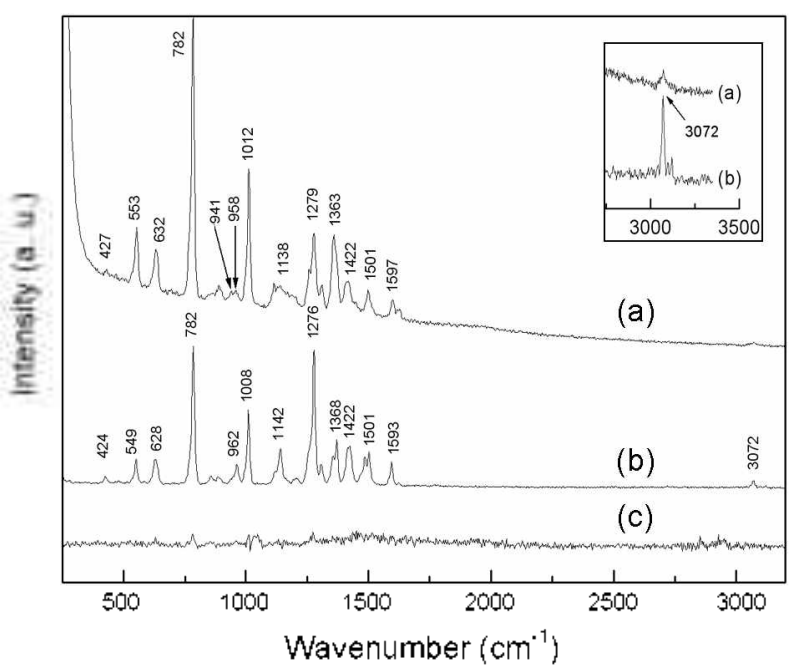

Figure 3. (a) SERS of BIZ $\left(1.0 \times 10^{-3} \mathrm{M}\right)$ and ordinary Ramarl spectra of (b) solid state and (c) $0.1 \mathrm{M}$ BL $Z$ solution in methanol.

adsorbed onto the metal, as also observed for other cationic species. ${ }^{2-3}$ The interaction of the protonated $\mathrm{N}$ atom of $\mathrm{BIZ}$ with $\mathrm{Cl}^{\prime}$ seems to be so strong that a CT occurs between the chloride and $\mathrm{BIZ}$ in acidic conditions. ${ }^{2}$ When the $\mathrm{H}_{2} \mathrm{SO}_{4}$ or $\mathrm{HNO}_{3}$ solution was used instead of $\mathrm{HCl}$. the SERS spectrum was not enhanced. But the SERS spectnum of the BIZ added to the $\mathrm{H}_{2} \mathrm{SO}_{4}$ and $\mathrm{KCl}$ solution was similar to Figure $5(\mathrm{a})$. These experimental results support the fonmation of a strong ionic pair. The enhancement of the band at $3072 \mathrm{~cm}^{-1}$ (Figure 5 . inset 
Table 1. Wavenumbers $\left(\mathrm{cm}^{-1}\right)$ and assignments corresponding to the calculated and experinental spectra of BIZ.

\begin{tabular}{|c|c|c|c|c|}
\hline $\begin{array}{c}\text { Calcul- } \\
\text { ation }\end{array}$ & $\begin{array}{l}\text { "Solid } \\
\text { Ramant }\end{array}$ & $\begin{array}{c}{ }^{a} \mathrm{SERS} \\
(\mathrm{pH}=7)\end{array}$ & $\begin{array}{c}{ }^{a} \mathrm{SERS} \\
(\mathrm{pH}=2)\end{array}$ & ${ }^{b}$ Assigmnent \\
\hline 425 & $424(w)$ & $427(\mathrm{vw})$ & - & $\delta(C-C-C) i p$ \\
\hline 556 & $549(\mathrm{~m})$ & $553(\mathrm{~m})$ & $550(\mathrm{~m})$ & $\delta(\mathrm{N}-\mathrm{H}) \circ o \mathrm{p}, \delta(\mathrm{N}-\mathrm{C}-\mathrm{N}) \circ$ \\
\hline 611 & $628(\mathrm{~m})$ & $632(\mathrm{~m})$ & $628(\mathrm{~m})$ & $\delta(\mathrm{C}-\mathrm{C}-\mathrm{C}) \mathrm{ip}$ \\
\hline 771 & $782(\mathrm{vs})$ & $782(\mathrm{vs})$ & $780(\mathrm{vs})$ & $\delta(\mathrm{C}-\mathrm{H})$ oop \\
\hline 951 & - & - & $942(\mathrm{~m})$ & $\mathrm{C}-\mathrm{C}$ ring breathing \\
\hline 956 & $962(w)$ & $958(\mathrm{vw})$ & - & $\delta(\mathrm{C}-\mathrm{H})$ oop \\
\hline 1000 & $1008(s)$ & $1012(s)$ & $1012(s)$ & $\delta(\mathrm{C}-\mathrm{C}-\mathrm{C}) 00 \mathrm{p}$ \\
\hline 1151 & $11+2(\mathrm{~m})$ & $1138(w)$ & - & $\delta(\mathrm{C}-\mathrm{H}) \mathrm{ip}$ \\
\hline 1151 & - & - & $1174(\mathrm{~m})$ & $\delta(\mathrm{C}-\mathrm{H}) \mathrm{ip}$ \\
\hline 1291 & $1276(s)$ & $1279(\mathrm{~m})$ & $1283(s)$ & $v_{\text {rng }}+\delta(\mathrm{C}-\mathrm{H}) \mathrm{ip}$ \\
\hline 1291 & $1309(\operatorname{sh})$ & $1309(\mathrm{sh})$ & - & $\delta(\mathrm{C}-\mathrm{H}) \mathrm{ip}$ \\
\hline 1408 & $1368(\mathrm{~m})$ & $1363(\mathrm{~m})$ & $1353(s)$ & $v(C=C)$ \\
\hline 1425 & $1422(\mathrm{~m})$ & $1422(\mathrm{~m})$ & - & $v(\mathrm{C}=\mathrm{C})$ \\
\hline 1495 & $1484(\operatorname{sh})$ & - & $1451(w)$ & $\delta(\mathrm{N}-\mathrm{H}) \mathrm{ip}$ \\
\hline 1495 & $1501(w)$ & $1501(w)$ & $1513(w)$ & $v(\mathrm{C}=\mathrm{C})$ \\
\hline 1607 & $1593(w)$ & $1597(w)$ & - & $v(\mathrm{C}=\mathrm{C})$ \\
\hline 1639 & $1622(\mathrm{vH})$ & $1627(\mathrm{vH})$ & $1624(w)$ & $v(\mathrm{C}=\mathrm{N})$ \\
\hline 3080 & $3072(w)$ & $3072(\mathrm{vw})$ & - & $v(\mathrm{C}-\mathrm{H})$ \\
\hline 3084 & $3118(\mathrm{vw})$ & - & - & $v(\mathrm{~N}-\mathrm{H})$ \\
\hline
\end{tabular}

$a_{s}=$ strong: $m=$ mediun; $w=$ weak: vw: very: vs = verv strong: $s h=$ shoulder; ${ }^{t} \mathrm{v}=$ stretching; $\delta=$ bending: ip $=$ in plane; $00 \mathrm{p}=$ out of plane. (a)

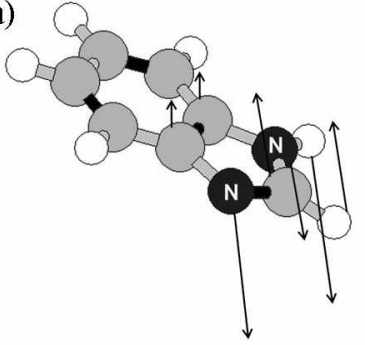

(c)

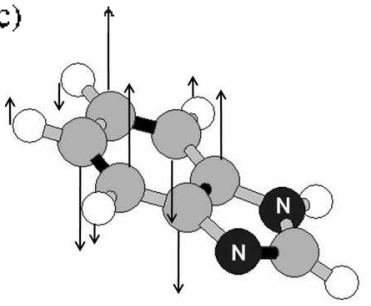

(b)

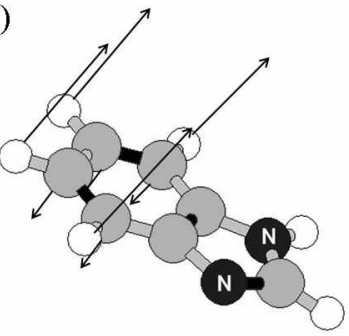

(d)

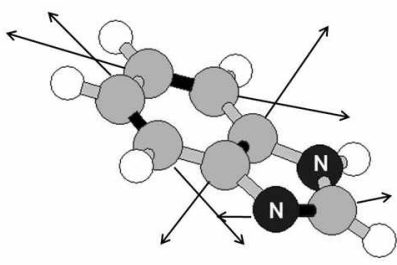

Figure 4. Vibrational modes of (a) 553 , (b) 782, (c) 1012 and (d) 1363 $\mathrm{cm}^{-1}$ for BIZ. The arrows represent the relative displacement of the nuclei for that specific vibrational mode.

spectra). which is assigned to the stretching mode of $\mathrm{C}-\mathrm{H}$. further supports the adsorption orientation as shown in Figure $6 \mathrm{~b}$. The SERS spectrum of BIZ revealed little enhancement at $\mathrm{pH}=12.0$ as shown in Figure $5 \mathrm{c}$. since the adsorption of molecules is supposed to be hindered by hydroxide ions previously adsorbed on to metal. It would rather similar to the ordinary Ramin spectrum of $0.1 \mathrm{MBIZ}$ solutions. In Figure 6 . the plausible adsorp-

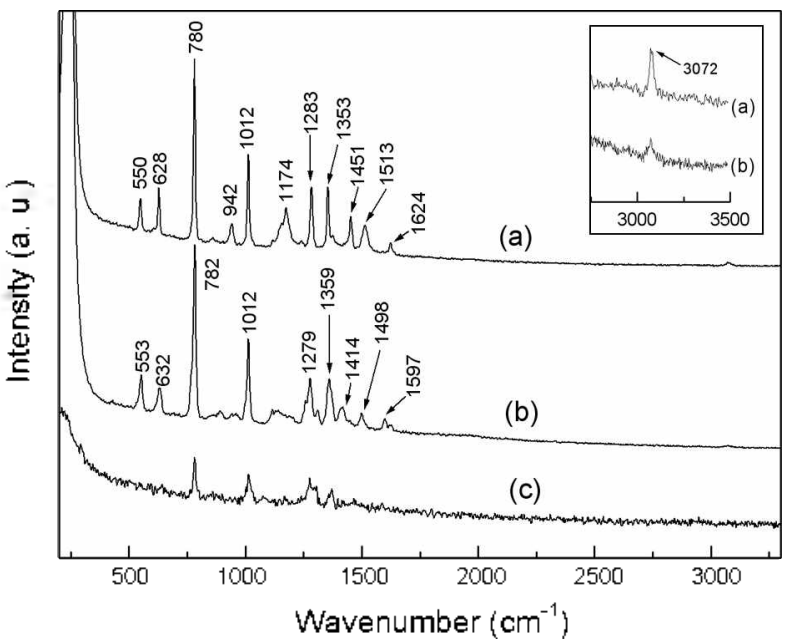

Figure 5. SERS spectra of $1.0 \times 10^{-3} \mathrm{M}$ benzimidazole on silver mirror at $($ a) $\mathrm{pH}=2.0$, (b) $\mathrm{pH}=7.0$, and (c) $\mathrm{pH}=12.0$.

(a)

(b)
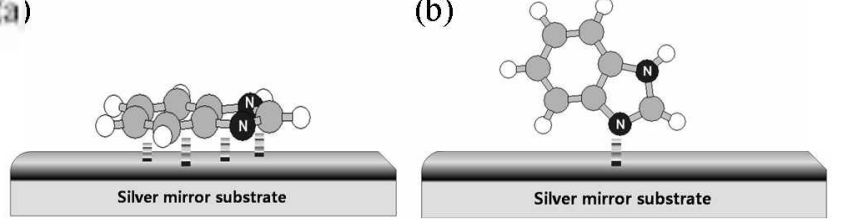

Figure 6. The plausible adsorption orientation of BIZ onto the silver mirror surface in (a) neutral and (b) acidic conditions.

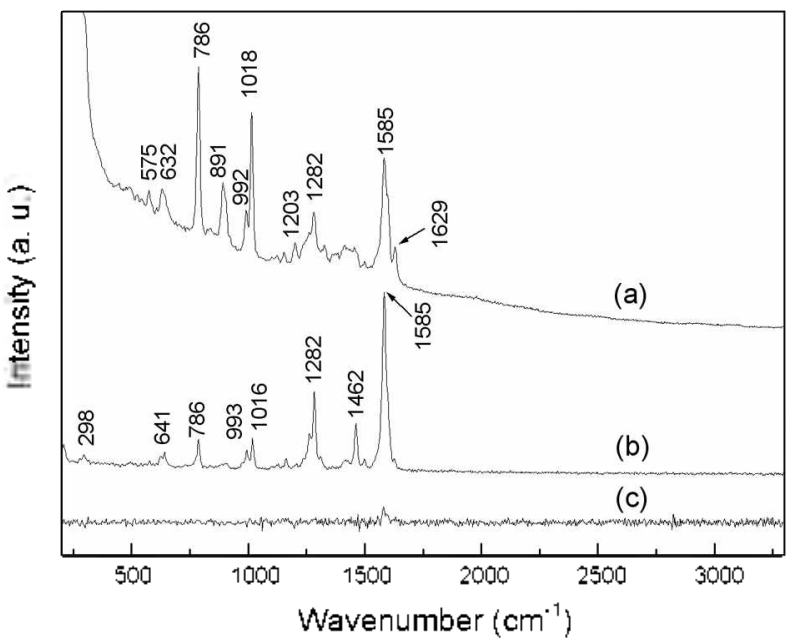

Figure 7. (a) SERS of TBZ $\left(10 \times 10^{-3} \mathrm{M}\right)$ and ordinary Raman spectra of (b) solid state and (c) $0.1 \mathrm{M} \mathrm{TBZ}$ solution in methanol.

tion orientations of BIZ onto the silver nurror surface in neutral and acidic conditions were presented.

Figure 7 shows the surface-enhanced Raman spectrum of TBZ adsorbed on silver nirror (a) and the ordinary Raman spectnum of TBZ in solid state (b) and $0.1 \mathrm{M}$ solution (c). The ordinary Rantan spectrum of TBZ solution also cannot be obtained as the same as BIZ. The most significant change in SER spec- 
Table 2. Wavenumbers $\left(\mathrm{cm}^{-1}\right)$ and assignments corresponding to the calculated and experimental spectra of TBZ.

\begin{tabular}{|c|c|c|c|c|}
\hline $\begin{array}{c}\text { Calcul- } \\
\text { ation }\end{array}$ & $\begin{array}{l}{ }^{3} \text { Solid } \\
\text { Raman }\end{array}$ & $\begin{array}{l}{ }^{3} \mathrm{SERS} \\
(\mathrm{pH}-7)\end{array}$ & $\begin{array}{l}{ }^{a} \mathrm{SERS} \\
(\mathrm{pH}-3)\end{array}$ & ${ }^{6}$ Assignment \\
\hline 298 & $298(w)$ & - & - & $\delta(\mathrm{C}-\mathrm{S}-\mathrm{C}) \mathrm{ip}$ \\
\hline 578 & - & $575(w)$ & $573(w)$ & $\delta(\mathrm{C}-\mathrm{C}-\mathrm{C}) \mathrm{ip}$ \\
\hline 635 & $641(w)$ & $632(w)$ & $641(w)$ & $\delta(\mathrm{C}-\mathrm{C}-\mathrm{C}) \mathrm{ip}, \partial(\mathrm{S}-\mathrm{C}-\mathrm{N}) \mathrm{ip}$ \\
\hline 771 & $786(\mathrm{~min})$ & $786(s)$ & $781(s)$ & $\delta(\mathrm{C}-\mathrm{H}) \mathrm{pop}$ \\
\hline 839 & - & $891(\mathrm{~m})$ & $889(\mathrm{mi})$ & $\delta(\mathrm{C}-\mathrm{C}-\mathrm{S}) \mathrm{oop}, \delta(\mathrm{C}-\mathrm{H}) \mathrm{oop}$ \\
\hline 988 & $993(\mathrm{sh})$ & $992(\operatorname{sh})$ & $987(\mathrm{~m})$ & $v(\mathrm{C}-\mathrm{S})$ \\
\hline 1010 & $1016(\mathrm{~m})$ & $1018(s)$ & $1015(s)$ & $\delta(\mathrm{C}-\mathrm{H})$ oop \\
\hline 1194 & - & $1203(w)$ & $1203(w)$ & $\partial(\mathrm{C}-\mathrm{H}) \mathrm{ip}$ \\
\hline 1297 & $1282(\mathrm{~s})$ & $1282(\mathrm{~m})$ & $1282(w)$ & $v_{\text {tum }}$ \\
\hline 1315 & & & $1 \hat{3} 1 \hat{3}(w)$ & $v(\mathrm{C}=\mathrm{C})$ \\
\hline 1362 & & & $1360(w)$ & $v(\mathrm{C}=\mathrm{C})$ \\
\hline 1434 & & $1415(\mathrm{vw})$ & $1432(w)$ & $v_{\text {touy }}$ \\
\hline 1451 & $1462(\mathrm{~m})$ & $1458(\mathrm{vw})$ & $1470(w)$ & $v(\mathrm{C}=\mathrm{N})$ \\
\hline 1584 & $1585(v s)$ & $1585(s)$ & $1601(\mathrm{~m})$ & $v_{\text {nIg }}+v(C=\mathrm{C})$ \\
\hline 1639 & & 1629(sh) & $1631(\mathrm{~m})$ & $v(\mathrm{C}=\mathrm{N})$ \\
\hline 3077 & & & $3086(v w)$ & $v(\mathrm{C}-\mathrm{H})$ \\
\hline
\end{tabular}

$a_{\mathrm{s}}=$ strong: $\mathrm{m}=$ medium: $\mathrm{w}=$ weak: $\mathrm{vw}=$ very: $\mathrm{vs}=$ very strong: $\mathrm{sh}=$ shoulder; " $v=$ stretching: $\delta=$ bending: ip $=$ in plane; $00 p=$ out of plane.

trum is the strong intensity decrease of the band 1585,1462 and $1282 \mathrm{~cm}^{-1}$. These are attributed to totally ring stretching vibrations as also confirmed by comparison with assignments made for similar structural pesticides. ${ }^{-299}$ While. the strong bands of intensity appearing at 786.1018 and $891 \mathrm{~cm}^{-1}$ in the SERS spectrum may be assigned to $\mathrm{C}-\mathrm{H}$ out-of plane bending modes and C-C-S out-of-plane bending mode. respectively. The band frequencies and their assignments based on literature and calculation are given in Table 2. . $1.30^{30}$ As shown in Figure 1. TBZ has delocalized $\pi$ electrons in the molecule of planar structure, since the benzimidazole and thiazole rings are not allowed to rotate. Therefore. according to the SERS selection rule. the strong intensities of these out-of-plane vibration modes in the SERS suggest the parallel orientation of TBZ molecules to the silver surface as shown in Figure 10a. Figure 8 presents diagrams of the nonmal mode vibration of 786,891. 1018 and 1585 $\mathrm{cm}^{-1}$ which are remarkable peaks in the SERS.

In other to compare the adsorption geometry according to the $\mathrm{pH}$. we obtained the SER spectra of TBZ at $\mathrm{pH} 2.0 .7 .0$ and 12.0 as shown in Figure 9. The spectral patterns of TBZ in acidic and neutral solutions were similar. Only the bands of 987 and $1631 \mathrm{~cm}^{-1}$ in the acidic SERS are enhanced a little which may be assigned to $\mathrm{r}(\mathrm{C}-\mathrm{S})$ and $\mathrm{v}(\mathrm{C}=\mathrm{N})$, respectively. Also. the $\mathrm{C}=\mathrm{C}$ and $\mathrm{C}=\mathrm{N}$ stretching modes are splitted slightly in $1200 \sim 1500$ $\mathrm{cm}^{-1}$ region. Therefore, most molecules were also adsorbed on surface via the delocalized $\pi$ electrons in planar rings and some molecules were adsorbed via a sulfur and nitrogen atoms tilted slightly to the silver surface. in acidic solutions. The enhancement of the band at $3086 \mathrm{~cm}^{-1}$ (Figure 9 . inset spectra), which is assigned to the stretching mode of $\mathrm{C}-\mathrm{H}$, further supports this adsorption orientation The plausible adsorption orientations of BTZ in neutral and acidic solutions were presented in Figure (a)

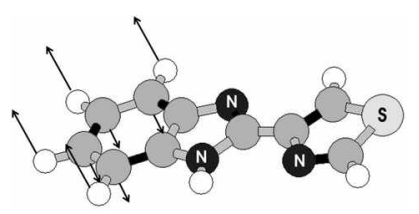

(b)

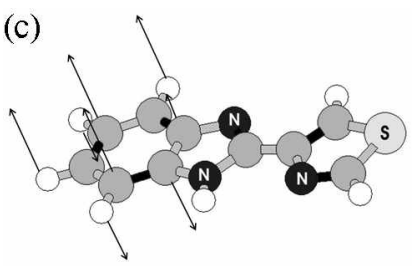

(d)
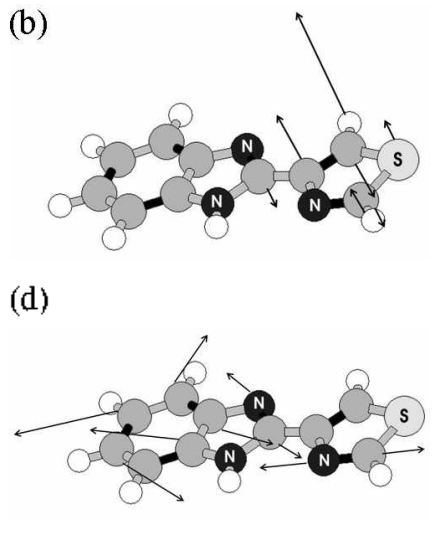

Figure 8. Vibrational modes of (a) 786, (b) 891 , (c) 1018 and (d) $1585 \mathrm{~cm}^{-1}$ for TBZ. The arrows represent the relative displacement of the nuclei for that specific vibrational mode.

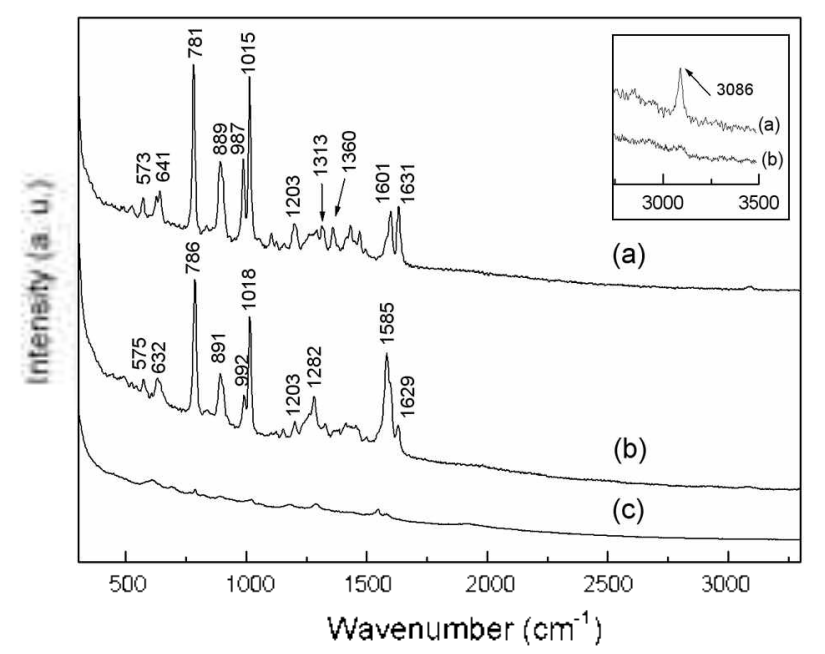

Figure 9. SERS spectra of $1.0 \times 10^{-3} \mathrm{M}$ TBZ on silver mirmor at (a) $\mathrm{pH}=$ 2.0 , (b) $\mathrm{pH}=7.0$, and (c) $\mathrm{pH}=12.0$.

(a)

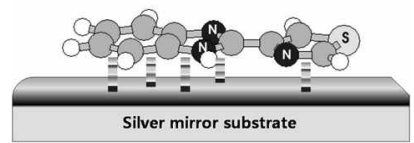

(b)

Figure 10. The plausible adsorption orientation of TBZ onto the silver mirror surface in neltral and acidic conditions.

10. The SERS spectrum of the basic solution of TBZ could not be enhanced as the same reason as BIZ.

\section{Conclusion}

SERS spectroscopy allows for the achievement of vibrational spectra of $1.0 \times 10^{-3} \mathrm{M} \mathrm{BIZ}$ and TBZ fungicide solutions. In SERS spectroscopy, there is no necessary preprocessing of 
sample solutions. therefore this technique could be employed for the detection of such fungicides in the environments. We have measured the SER spectra of BIZ and TBZ adsorbed on silver mirror substrates. In case of BIZ. completely different adsorption mechanisms are possible depending on the $\mathrm{pH}$ conditions. In neutral conditions, the adsorption of BLZ occurs $v a$ delocalized $\pi$ electrons parallel to the surface and in acidic conditions. the interaction of the protonated $\mathrm{N}$ atom with chloride ion lead to perpendicular adsorption to the surface. The adsorptions of TBZ have similar mechanisms. Most adsorptions occur parallel to the surface via the delocalized $\pi$ electrons in planar rings but some adsorptions are slightly tilted via the sulfur and nitrogen atoms in acidic conditions. In basic conditions. the SER spectra of both of BIZ and TBZ could not be obtained since the adsorption of molecules is supposed to be hindered by hydroxide ions.

Acknowledgments. This work was supported by Korea Research Foundation Grant No. KRF-2006-005-j02401 and by Kyungpook National University Research Fund, 2007 (KRF$2004-005-\mathrm{c}-00006$ ).

\section{References}

1. Tames, D. M:; Gilles, H. M. Human Antiparasitic Dnigs: Pharmacologv and tisage: Jolu Wiley \& Sons: New York, 1996: $p$ 206

2. Delescluse, C.; Piechock, M. P : Ledirac, N.; Hines, R. H.; Li, R.; Gidrol X.: Ralunani, R, Biochem. Phamacol. 2001, 61, 399.

3. Lezcano, M.: Soufi, W. A. L.: Novo, M. Rodriguez-Nunez, E.; Tato, I. V. J.Agric. Chem. 2002, 50, 108

4. Frenich, A. G.; Zamora, D. P.; Martinez Vidal, T. L.; Martinez Galera, M. M. Anatyitica Chinica Acto 2003, $+47,211$.

5. Lombardi, M.; Baschini, M.: Tores Sanchez, R. M. Applied Clon: $S_{c i .} \mathbf{2 0 0 3}, 2+4,43$.

6. Garcia-Reves, I. F; Llorent-Martinez, E. J.; Ortega-Barrales, P: Molina-Diaz, A Analytica Chimica Acta 2006, 557, 95.
7. Frenich, A. G.; Zamora, D. P. Martinez Vidal, J. L.; Galera, M. M. Analutical Chimica Acta 2003, $\$ 77,211$.

8. Sundaraganesan, N.; Ilakiamani, S.; Subramani, P, Dominic Toshua, B. Analutical Chimica ticta Part $A$ 2007, 67,628.

9. Albrecht, M. A.; Creighton, J. A. J. Am. Chem. Soc. 1977, 99 , 5215 .

10. Moskovits, M. J. Chem. Phus. 1978, 69, 4159

11. Adrian, F. J. Chent. Phys Lett 1981, 78, 45.

12. Wang. D. S.: Kerker, M. Phss. Rev. 1 1981, 24, 1777.

13. Joo, S. W.; Han, S. W: Kim, K. J. Colloid hnterface Sci. 2001 , 240,391

14. Jung, Y. M.; Lim, T. W.; Kim, E. R.; Lee, H.; Lee, M. S. Bull. Konew Chem. Soc. 2001, 22,318.

15. Jeanette, G. G.: Cook, C.: Koglin, E. J. Raman Spectrosc. 1993, 24,609

16. Mukherjee, K.; Sanchez-Cortes, S.; Garcia-Ramos, J. V. Iibrational Spectroscopy. 2001, 25, 91.

17. Saito, Y.; Wang, J. T.; Smith, D. A.; Batchelder, D. N. Langnuir 2002, 28,2959

18. Lee, C. J-; Kang, T. S.; Kim, M. S.; Lee, K. P.; Lee, M. S. Buhl. Konew Chem. Soc. 2004, 25(8), 1212.

19. Lee, C. I.: Lee, S. Y,; Karim, M. R.; Lee, M. S. Spectrochimica Acta Pant $2007,68,1313$.

20. Wingrove, A. S.: Caret, R. L. Organic Chemismy: Haper \& Row Publishers: London, 1981; p 185.

21. Socrates, G. Infrored and Ramtan Characteristic Group Frequencies: John Wiley \& Son, Ltd: 2001: p 229

22. Sundaraganesan, N.; Ilakiamani, S.; Subramani, P.; Dominic Toshua, B. Spectrochimica tcta Pait t 2007, 67,628.

23. Ni, F.; Cotton, T. M. J. Ramton Spectrosc. 1988, 19, 429.

24. Lee, H. I.; Sul, S. W.; Kim, M. S. J. Raman Spectrose. 1988,19 , 491.

25. Millan, J. I.; Garcia-Ramos, J. V.; Sanchez-Cortes, S.; RodriguezAmaro, R. J. Ramam Spectrosc. 2003, 3†, 227.

26. Lévi, G.: Pantigny, I.: Marsault, I. P., Aubard, I. J. Raman Spectrose $1993,24,745$

27. Oh, S. T.: Kim, K.: Kim, M. S. J. Phus Chem 1991, 51, 8844.

28. Monk, P. S ; Hodgkinson, N. M. Electrochim Acta 1998, $43,245$.

29. Forster, M.: Girling, R. B.; Hester, R. E. J. Raman Spectrosc. 1982 , $12,36$.

30. Lopez-Ramirez, M. R.; Guerrini, L.; Garcia-Ramos, J. V.; San1chez-Cortes, S. Tibrational Spectroscopy 2008, $\$ 8,58$. 